\title{
ASESORAMIENTO PROFESIONAL Y MANEJO DE NUEVAS TECNOLOGÍAS EN UNIDADES DE PRODUCCIÓN HORTÍCOLAS DEL GRAN LA PLATA, ARGENTINA
}

\author{
RELATIONSHIP BETWEEN PROFESSIONAL ADVICE AND USE OF NEW \\ TECHNOLOGIES IN HORTICULTURAL FARMS OF LA PLATA, ARGENTINA
}

\begin{abstract}
ASSESSORAMENTO PROFISSIONAL E MANEJO DE NOVAS TECNOLOGIAS EM UNIDADES DE PRODUÇÃO HORTÍCOLAS DA GRANDE LA PLATA, ARGENTINA
\end{abstract}

\author{
Ramón Isidro CIEZA
}

RESUMEN

\begin{abstract}
En el periodo 1990-2001 se han dado modificaciones en el sector productor de hortalizas del Gran La Plata, Argentina a partir de la adopción del cultivo bajo cubierta y un conjunto de tecnologías complementarias. El grado de incorporación de estas nuevas tecnologías y su éxito productivo-comercial ha sido heterogéneo de acuerdo al tipo de productor y sus posibilidades de adaptación a estos cambios. La hipótesis es que aquellos productores que adoptaron nuevos paquetes tecnológicos en forma exitosa, desarrollando un perfil empresarial más nítido han contado con apoyo técnico de un profesional. El objetivo perseguido en este trabajo es conocer la heterogeneidad en cuanto al manejo tecnológico-comercial de las unidades productivas hortícolas y la incidencia del asesoramiento técnico dentro de ellas. Durante los meses de febrero a mayo de 2004 se realizaron 30 encuestas a horticultores en tres zonas representativas del Cinturón Hortícola de La Plata. Se determinaron 10 variables ( 4 propias del productor y 6 referidas a la producción-comercialización) que fueron sometidas a técnicas multivariantes y bivariantes de ordenamiento y clasificación. Se identificaron tres grandes grupos de unidades productivas hortícolas en función de las tecnologías utilizadas, las formas de acceso al conocimiento técnico y los canales de comercialización utilizados. No se encontró vinculación entre las variables propias del productor y el manejo técnicocomercial de la unidad productiva. Se concluye que los asesores técnicos han influido favorablemente en la adecuación de los nuevos modelos tecnológicos, con mayor preponderancia en planteos empresariales.
\end{abstract}

Palavras-chave: Horticultura; Nuevas tecnologías; Asesoramiento técnico; Análisis multivariado.

\begin{abstract}
Between 1990 - 2001, in the horticultural sector of La Plata, Argentina, important changes was taken place because the adoption of greenhouse cultivation and a group of complementary technologies. The incorporation degree of these new technologies and their productive commercial success was variable according to the farmers type and their possibilities of adaptation to these changes. The hypothesis is that those farmers that adopted successfully new technological packages, developing a best managerial profile, have had professional advice. The aim of this work is to study the technological-commercial heterogeneity of the horticultural farms and the incidence of the technical advice in this heterogeneity. 30 surveys to horticultural farmers were carried out between February and May of 2004 in three representative sections of the Horticultural Area of La Plata, Argentina. 10 variables were determined (4 about each producer and 6 in relationship with their production - commercialization ways). This variables were analyzed using multivariate and bivariate statistical methods. Three groups of horticultural farms were identified according to the type of technologies used, the way to access to technical knowledge and the utilized commercialization channels. Relationships between farmers characteristics and the technical-commercial management of each farm was not found. We concluded that technical advisers have favorably influenced in the adaptation of the new technological models, preponderantly in managerial farmers.
\end{abstract}

Key-words: new technologies; technical advice; multivariate analysis.

\section{RESUMO}

No período de 1990-2000 ocorreram modificações no setor produtivo de hortaliças da Grande La Plata, Argentina a partir da adoção de cultivo protegido e de um conjunto de tecnologías complementares. Ograu de incorporação destas novas tecnologías e seu êxito produtivocomercial tem sido heterogêneo conforme o tipo de produtor e suas posibilidades de adaptação a estas mudanças. A hipótese é que aqueles produtores que adotaram novos pacotes tecnológicos com êxito, desenvolvendo um perfil empresarial mais nítido, tem contado com o apoio técnico de um profissional. O objetivo buscado neste trabalho é conhecer a heterogeneidade quanto ao manejo tecnológico-comercial das unidades produtivas hortícolas e a incidência do assessoramento técnico dentro delas. Durante os meses de fevereiro a maio de 2004 se realizaram 30 sondagens a horticultores em tres zonas representativas do Cinturão Hortícola de La Plata. Se determinaram 10 variáveis (4 próprias do produtor e 6 referidas a produção-comercialização) que foram submetidas a técnicas multivariantes e bivariantes de ordenamento e classificação. Se identificaram tres grandes grupos de unidades produtivas hortícolas em função das tecnologías utilizadas, das formas de acesso ao conhecimento técnico e dos canais de comercialização utilizados. Não se encontrou vinculação entre as variáveis próprias do produtor e o manejo técnicocomercial da unidade produtiva. Conclui-se que os assessores técnicos tem influído favoravelmente na adequação dos novos modelos tecnológicos, com maior preponderância em estabelecimentos empresariais.

Palavras Chave: Horticultura; Novas tecnologías; Assessoramento técnico; análise miltivariada.

${ }^{1}$ Ing. Agr. (Ms. Sc.). Comision de Investigaciones Cientificas de la Provincia de Buenos Aires, Argentina. Becario de Perfeccionamiento. Facultad de Ciencias Agrarias y Forestales. Departamento de Desarrollo Rural. 60 y 119 La Plata (CP 1900) Provincia de Buenos Aires. Argentina.E-mail cieza@ceres.agro.unlp.edu.ar. 


\section{INTRODUCCIÓN}

El Cinturón Hortícola del Gran La Plata comprende el área productiva de mayor envergadura del Cinturón Hortícola Bonaerense. Este último abastece de verduras frescas a uno de los núcleos poblacionales mas densos de la Argentina, Ciudad Autónoma de Buenos Aires y el Conurbano Bonaerense, con una población de alrededor de 10 millones de personas. El Cinturón Hortícola del Gran La Plata cuenta con 593 unidades de producción hortícolas, cubriendo un total de 6154,34 hectáreas. De estas, aproximadamente el $60 \%$ poseen cultivos bajo cobertura ${ }^{2}$, con una totalidad de 622,74 hectáreas bajo esta modalidad [1]. Esta región ha experimentado intensos cambios en el periodo 1990-2001, en consonancia con el periodo de convertibilidad y el ingreso de tecnologías importadas de los países centrales a bajo precio [2]). Simultáneamente se desarrollaron cambios en los circuitos de comercialización a partir de la irrupción de las primeras cadenas de hipermercados, con nuevas técnicas de presentación de productos y mayor exigencia de calidad que traccionaron en las formas de producción empresarial hortícola [3]). La etapa del invernáculo, se inicia a principios e los '90 con la difusión del cultivo bajo cobertura y tecnologías asociadas a este. Su amplia utilización se atribuye a la minimización de los efectos climáticos generando una mayor factibilidad de cosechar productos de calidad comercial y su obtención en forma anticipada a los obtenidos convencionalmente; con lo cual en la mayoría de las especies el precio era significativamente mayor [4].

La incorporación de estas innovaciones ha requerido de la apropiación de nuevas habilidades en pos de un manejo armónico del sistema productivo. En esta instancia el asesoramiento técnico y otras formas de acceso al conocimiento habrían repercutido positivamente en la adecuación de los modelos tecnológicos a las nuevas formas de produccióndistribución y consumo en el sector de productos frescos.

La hipótesis de trabajo es que aquellas Unidades de Producción que adoptaron el cultivo bajo cobertura aplicando nuevas innovaciones asociadas a este, que desarrollaron canales diferenciales de comercialización, planificación de la producción a lo largo del tiempo y eficiencia en el manejo económico de la empresa, se encuentran asesorados técnicamente por un profesional de la Ciencias Agrarias.

La producción hortícola regional cuenta con una alta heterogeneidad de productores $[5,6]$ en cuanto a grado de capitalización, organización social del trabajo, superficie utilizada, cultivos producidos, canales de comercialización, entre otros. Del mismo modo se producen diferencias en cuanto a las formas de acceso al conocimiento para el manejo de los aspectos tecnológicos de la unidad de producción.

En virtud de esta complejidad se adquiere el
Enfoque de Sistemas [7]) como marco conceptual y metodológico de la investigación en cuestión. Este enfoque, en el marco de la teoría de la modernización, consta con una prolongada tradición de trabajos en el INTA $^{3}$, siendo de utilidad para diferenciar los distintos Sistemas Reales de Producción (SRP) por su comportamiento a la incorporación de tecnología, identificando los factores que limitan o retardan el proceso de adopción. Esta visión considera al predio como un sistema social-económico de producción, dado que los elementos técnicos, económicos y sociales que la caracterizan interactúan con todo el sistema social, en particular con su contexto socioeconómico. Estos elementos sean endógenos o exógenos a la unidad productiva, tienen una razón de causalidad sobre su producción y por ende sobre su nivel tecnológico (Ibíd.).

El objetivo de este trabajo es conocer la heterogeneidad en el manejo tecnológico-comercial de las distintas unidades productivas hortícolas y la incidencia del asesoramiento técnico en las estrategias productivas para la permanencia dentro del mercado formal de productos frescos. En este sentido se busca validar cuantitativamente conceptualizaciones que surgieron en trabajos cualitativos desarrollados previamente por este autor. Se reconocen otros condicionantes de los cambios técnicos, pero por razones de precisión de análisis se ha recortado el análisis en algunos hipotéticos condicionantes.

\section{MATERIALES Y MÉTODOS}

Se elaboró una encuesta para relevar la información utilizada en la construcción de las variables. A partir de las caracterizaciones realizadas por Hang et al, [3], Benencia, [8] y la información disponible en el Censo hortícola 1998, se trabajó durante el año 2003 con una muestra representativa de 30 productores hortícolas del distribuidos en tres zonas con fuerte tradición de producción hortícola del Cinturón Hortícola Platense: Arana, Colonia Urquiza y Gorina. Se identificaron variables propias del productor y otras variables referidas a la produccióncomercialización, estas son: años en la producción, origen, generación de productores, educación formal, superficie cubierta, relación sup. campo/sup. cubierta, innovaciones adoptadas, asesor, gestión y comercialización. Estas variables fueron seleccionadas en función de entrevistas en profundidad a 4 productores y 11 técnicos del Cinturón Hortícola Platense durante los meses de noviembre y diciembre de 2002, y un posterior análisis cualitativo realizado por este autor donde se desprende la hipótesis de trabajo [9].

Se procedió al archivo de los datos de las encuestas confeccionándose una serie de escalas con el objetivo de efectuar una clasificación de las diversas situaciones detectadas. Se cuantificó cada una de las variables que sirvieron para caracterizar los 
componentes conceptuales de los distintos establecimientos, asignándole una escala de valores para su tratamiento estadístico:

\section{Descripción de las variables y asignación de la escala}

\section{i) Variables propias del productor}

a) Años en la producción: Se determinó los años que se encontraba en la producción estableciéndose una escala de 1 a menos de 15 años, 2 entre 15 y 30 y 3 a mas de 30 años.

b) Origen: Se evaluó por medio de una escala cual fue el origen del productor encuestado, asignándose un valor de 1 en el caso que hubiera sido mediero o asalariado hortícola (ex empleados hortícolas), 2 en el caso que provenga de una producción familiar (hijo o nieto de productor) y un valor de 3 si correspondía a un productor que provenga de una actividad distinta a la hortícola.

c) Generación de productores: Se analizó la generación en la que correspondían los productores. Se asignó un valor de 1 a aquellos que eran primera generación de productores; un valor de 2 a los que eran segunda generación y un valor de 3 los que eran tercera o cuarta generación.

d) Educación Formal: Se estableció una escala del grado de escolaridad alcanzado, siendo 1 a estudios primarios; 2 a estudios secundarios, y 3 a estudios terciarios; no distinguiendo si estos eran completos o no.

ii) Variables productivas- comerciales

e) Superficie Cubierta: Por medio de una escala se evaluó la superficie bajo cobertura plástica con un valor de 0 a aquellos que tuvieran entre 0 y 0,25 has bajo cubierta, 1 entre 0,26 y 1,25 hectárea, 2 entre 1,26 y 2,99 hectáreas y 3 entre 3 y 10 hectáreas de cultivo bajo cubierta.

f) Relación Superficie a campo/ Superficie bajo cubierta: Mediante una escala se evaluó los valores de esta relación. Se estableció un valor de 3 a aquellos establecimientos con valores entre 0 y 0,5 ; un valor de 2 entre 0,6 y 3,5; un valor de 1 entre 3.6 y 10 ; un valor de 0 para aquellos de mas de 10 .

g) Cantidad de innovaciones: Mediante una escala se identificó de acuerdo a las innovaciones de última generación ${ }^{4}$ incorporadas en el predio: 0 ninguna innovación, 1 para 1 innovaciones, 2 para 2 innovaciones y el valor de 3 para 3 o mas innovaciones.
h)Asesor: Se determino si la unidad productiva contaba con asesoramiento técnico formal, esto es una visita de un profesional de las Ciencias Agrarias en un lapso de 15 días como mínimo, correspondiendo un valor de 1 en caso positivo y 0 en caso negativo.

i) Gestión: Se evaluó la realización de una gestión económica en la unidad productiva correspondiendo un valor de 1 en caso positivo y 0 cuando esto no ocurre.

j) Comercialización: Se estableció una escala de acuerdo al grado de involucramiento del productor en la comercialización, la diferenciación de estos canales de venta y las posibilidades de obtención de sobreprecios. Esto es 0 en canales poco diferenciados y de mayor número de intermediarios; 1 canales medianamente diferenciados con un mayor involucramiento del productor a esta etapa; 2 canales de alto involucramiento del productor en mercados, 3 canales de alta exigencia del productor en logística, gestión de la comercialización y poder de negociación (Grandes centros de compra como hipermercados)

Los datos fueron analizados por técnicas de análisis multivariado, incluyendo un análisis de agrupamiento "clusters análisis" y análisis de componentes principales (ACP) siguiendo una secuencia mixta de técnicas que incluyen ${ }^{5}$ :

a) Obtención de una matriz básica de 30 productores por 10 variables

b) Obtención de un fenograma de distancia entre productores ${ }^{6}[10]$

c) Obtención de una matriz de correlación entre variables $(10 \times 10)$ a partir del coeficiente de momento o producto de correlación de Pearson [11].

d) Análisis transversal bivariado para relacionar individualmente las variables relevantes con el asesoramiento técnico.

\section{RESULTADOS}

De las 30 unidades productivas relevados 16 (53.3\%) contaban con asesoramiento técnico. En función del análisis de la correlación ${ }^{7}$ (Tabla 1) se visualiza una baja asociación entre las variables propias del productor (variables i) y las variables productivas- comerciales (variables ii). Del mismo modo no se observan asociaciones entre si en las variables propias del productor (variables i).

${ }^{2}$ Comprende la producción de hortalizas dentro de invernáculos, estructuras permanentes o desarmables con cobertura transparente, dentro de las cuales se realiza todo el ciclo del cultivo.

${ }^{3}$ Instituto Nacional de Tecnología Agropecuaria. Organismo estatal de generación y transferencia de tecnología de la Republica Argentina.

${ }^{4}$ Se considera innovación de última generación a aquella que no se encontraba disponible en forma masiva en el mercado hortícola argentino antes de 1990 (son fertirriego a partir del riego por goteo, cabezal de riego computarizado, utilización de monitoreo de plagas y utilización de híbridos de alto potencial de rendimiento, compra de plantines controlados, control de poscosecha, nuevas formas de conducción del cultivo.). 
CIEZA, R.I. Asesoramiento profesional y manejo...

TABLA 1 - Análisis de correlación entre 10 variables de 30 productores del Cinturón Hortícola Platense ${ }^{8}$.

\begin{tabular}{|c|c|c|c|c|c|c|c|c|c|c|}
\hline & A_OS & ORIGEN & GENERACI & FORMACIO & CUB & RELAC_CA & INNOVACI & ASESOR & GESTI_N & COMER \\
\hline A_OS & 1.00 & -.10 & 18 & -.35 & -.06 & .19 & -.27 & -.24 & -.07 & .03 \\
\hline ORIGEN & -.10 & 1.00 & .37 & .27 & .32 & .15 & .58 & .39 & .45 & .17 \\
\hline GENERACI & .18 & .37 & 1.00 & .18 & .37 & .15 & .28 & .12 & .24 & -.20 \\
\hline FORMACIO & -.35 & .27 & .18 & 1.00 & .08 & .02 & .33 & .17 & .29 & .01 \\
\hline SUP CUB & -.06 & .32 & .37 & .08 & 1.00 & .65 & .72 & .66 & .43 & .34 \\
\hline RELAC_CA & .19 & .15 & .15 & .02 & .65 & 1.00 & .61 & .74 & .40 & .49 \\
\hline INNOVĀCI & -.27 & .58 & .28 & .33 & .72 & .61 & 1.00 & .66 & .52 & .24 \\
\hline ASESOR & -.24 & .39 & .12 & .17 & .66 & .74 & .66 & 1.00 & .63 & .50 \\
\hline GESTI_N & -.07 & .45 & .24 & .29 & .43 & .40 & .52 & .63 & 1.00 & .32 \\
\hline COMER & .03 & .17 & -.20 & .01 & .34 & .49 & .24 & .50 & .32 & 1.00 \\
\hline
\end{tabular}

Fuente: Elaboración propia en base a encuesta realizada, 2003.

Las variables productivas- comerciales se encuentran correlacionadas a excepción de la variable comercialización con la realización de gestión y la cantidad de innovaciones incorporadas en el predio. Se advierte un alto nivel de significancia en la correlación para las variable asesor (presencia de asesoramiento técnico) con superficie cubierta, relación superficie campo/cubierta, cantidad de innovaciones incorporadas, gestión de la unidad productiva, y canales diferenciales de comercialización.
A partir de un análisis bivariado, se observa una fuerte asociación entre el asesoramiento técnico en el predio y la superficie bajo cubierta (Figura 1). De la totalidad de predios que realizaban una gestión económica- financiera y planificación del predio productivo el 91,7 \% contaba con asesoramiento técnico. Cuando se realiza un análisis bivariado vinculando la forma de comercialización a la existencia de un asesor profesional se encuentra una fuerte relación entre asesoramiento y canales de mayor complejidad de venta (Figura 2).

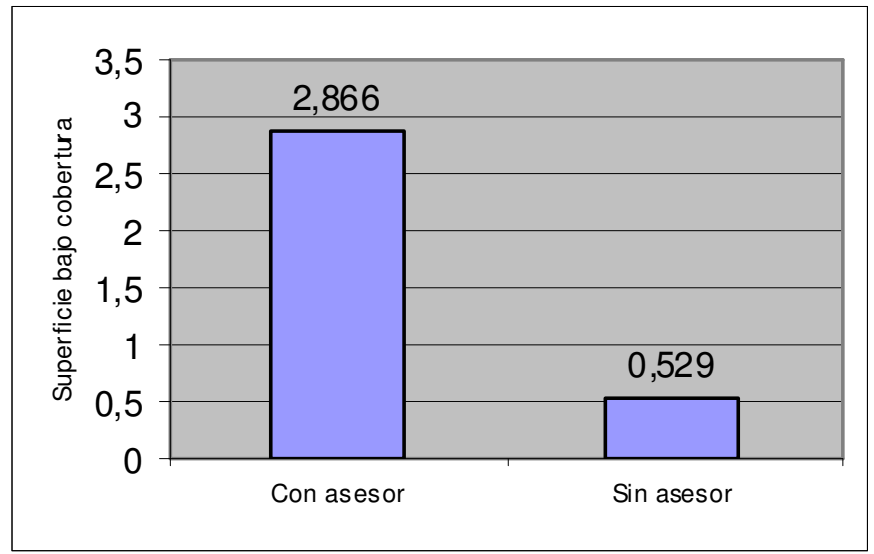

Fuente: Elaboración propria en base a encuesta realizadas (2003).

FIGURA 1 - Superficie media bajo cubierta en unidades productivas -con y sin asesoramiento técnico- en el Cinturón Hortícola Platense, Argentina.

\footnotetext{
${ }^{5}$ Para el tratamiento estadístico se utilizó el programa "statistica".

${ }^{6}$ El análisis de agrupamiento comprende técnicas que a partir de reglas arbitrarias forman grupos de objetos que se asocian por su grado de similitud (CRISCI y LOPEZ AMENGOL, 1983).

7 La correlación esta asociada a técnicas descriptivas por lo que nos dice algo sobre una relación conjunta entre variables (STELL y TORRIE, 1988: 268).

${ }^{8}$ Marked correlations are significant at $p<.05000 \mathrm{~N}=30$ (Casewise deletion of missing data). Las correlaciones mayores de 0.361007 son significativas.
} 


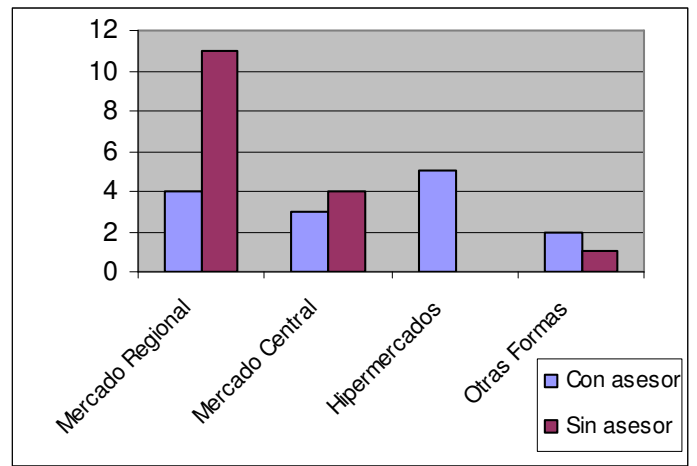

Fuente: Elaboración propria en base a encuesta realizadas (2003).

FIGURA 2 - Canales de comercialización de unidades productivas con o sin asesoramiento técnico -en cantidad-.

A partir del agrupamiento realizado por el fenograma (Figura 3) proveniente de la matriz de datos originada a partir del análisis de componentes principales sobre la matriz de distancia, se identificaron tres grandes grupos de productores:

El grupo A corresponde a productores con predominancia del cultivo bajo cubierta, y en algunos casos con toda la superficie productiva bajo esta modalidad. Estos productores en su gran mayoría se encuentran asesorados técnicamente. Su producción esta altamente especializada en pocos cultivos que venden en canales diferenciales de venta.

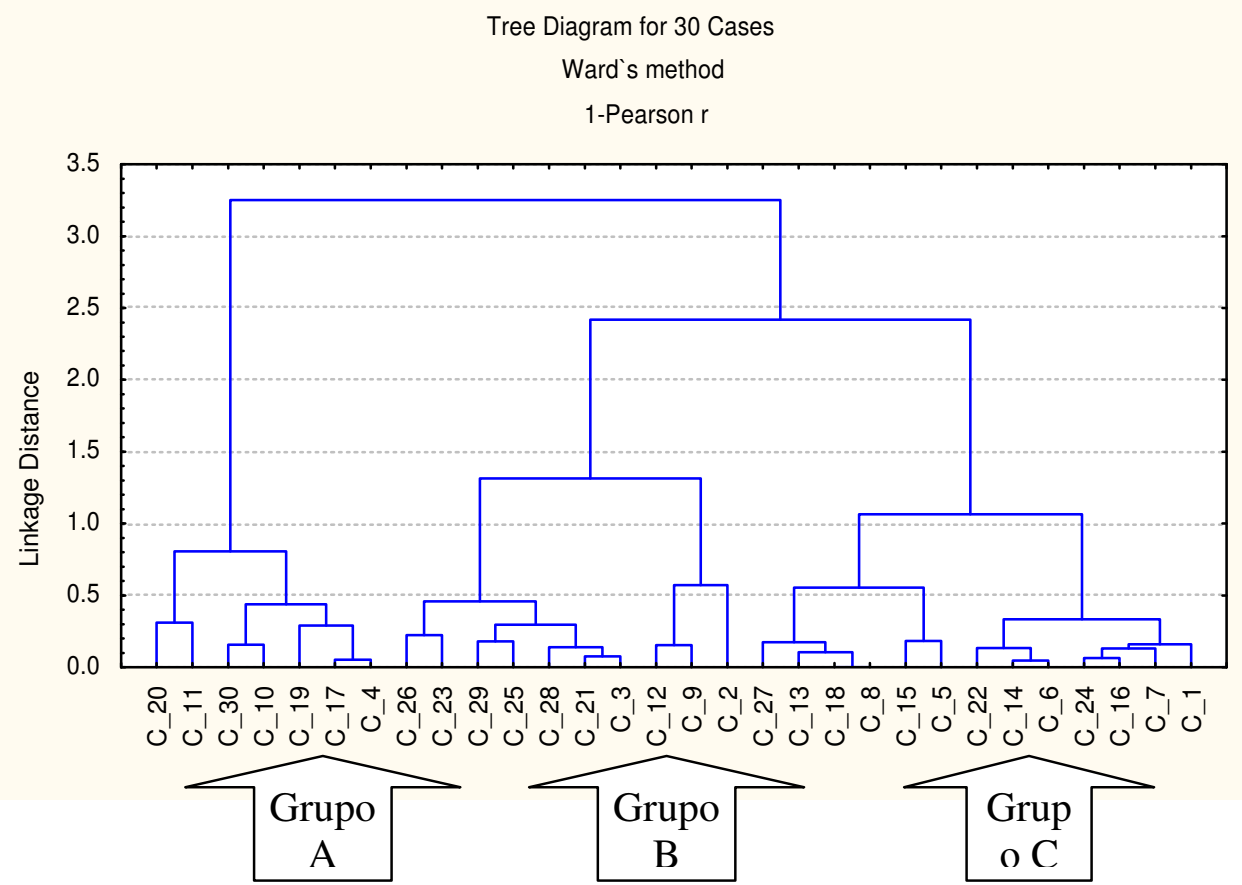

Fuente: Elaboración propria en base a encuesta realizadas (2003).

FIGURA 3 - Fenograma de 30 productores en base a 10 variables del Cinturón Hortícola Platense.

En cuanto al nivel tecnológico se considera que cuentan con una mayor cantidad de innovaciones, distanciándose de los restantes productores en lo que respecta a su uso.

El grupo B pertenece a productores con superficies equiparadas de cultivo a campo y bajo cobertura, aunque con predominio de las primeras en cuanto a superficie. Estos productores se caracterizan por una producción diversificada a campo, especializándose en solo algunos productos en invernáculo. En general comercializan en mercados concentradores a través de consignatarios o puestos de playa libre. En gran parte de ellos existe la presencia de un asesor técnico orientado hacia los cultivos bajo cubierta y eventualmente sobre los cultivos a campo. Su nivel tecnológico para los cultivos bajo cobertura es de medio a alto, y en el caso de los cultivos a campo realizan un manejo tradicional.

El grupo C corresponde a productores tradicionales donde la producción se basa 
fundamentalmente en cultivos a campo, pudiendo en algunos casos tener una pequeña porción de cultivo bajo cubierta. La producción es diversificada y orientada a los mercados concentradores locales a través de puestos de playa libre o venta en la quinta a intermediarios. En su gran mayoría no cuentan con asesores evacuando las dudas técnicas a través de las casas proveedoras de insumos o vecinos. El manejo de la unidad de producción es típicamente tradicional, con bajos niveles de inversión por unidad de superficie.

\section{DISCUSIÓN}

El comienzo del cultivo bajo cobertura y las exigencias de calidad de nuevos canales de comercialización modificaron radicalmente las formas de producción convencional hortícola, necesitando de un cambio en el manejo de los sistemas productivos; en este sentido los técnicos jugaron un papel preponderante. La complejidad que significó el manejo de nuevas tecnologías produjo que los principales agentes que mejor interpretaran los nuevos sistemas productivos fueran los asesores técnicos y en menor medida los productores y medieros.

El acceso a nuevos conocimientos a partir de jornadas técnicas, acceso a bibliografía especifica, redes de dialogo técnico con otros profesionales; la visión de varias unidades productivas en forma simultanea y la educación universitaria los posicionó en un lugar privilegiado para el manejo de las nuevas tecnologías asociadas al cultivo bajo cobertura (Cieza, op. cit).

Las políticas neoliberales implantadas en la Argentina en la década del 90 produjeron un retiro parcial en cuanto a adaptación y transferencia tecnológica de los organismos del estado, como contrapartida el asesoramiento privado y las empresas proveedoras de insumos tomaron preponderancia en el manejo de las nuevas tecnologías. En aquellas unidades productivas con asesoramiento privado, los técnicos se consolidaron en el manejo del paquete tecnológico a partir de recomendaciones técnicas y de organización de los factores de producción. En las unidades productivas con predominancia del cultivo a campo el manejo tecnológico continuó en manos del productor y el asesoramiento externo se tornó coyuntural a partir de consultas puntuales a técnicos asociados a empresas proveedoras de insumos.

La gestión económica-financiera y la planificación en el mediano o largo plazo a partir de registros de producción, de productos químicos utilizados, de control de la verdura cosechada y/o de precios obtenido de los productos queda supeditado casi exclusivamente a los planteos empresariales de alto nivel tecnológico bajo asesoramiento técnico (grupo A). Aquellos productores que venden sus productos en canales más complejos, de mayor exigencia de calidad y de organización en la entrega del producto (como el caso de hipermercados) cuentan, en general, con un profesional que los asesora técnicamente. El nivel educativo no se vincula a la estrategia productiva-comercial del sistema productivo, no contando con un patrón común de referencia en cuanto a esta variable el grupo de productores empresariales de alto nivel tecnológico. Del mismo modo los años y la cantidad de generaciones en la producción no se asocia al manejo tecnológico-comercial que realiza en su unidad productiva, por lo que productores con similares trayectorias productivas ocupan lugares distintos en las tipologías presentadas.

\section{CONCLUSIONES}

Se considera que el proceso de adopción tecnológica no es lineal, ni unívoco producto de una reacción estimulo- respuesta; por lo tanto no se puede aseverar que estuvo subordinado exclusivamente a la variable asesoramiento técnico. Sin embargo se demuestra una fuerte asociación entre esta variable y aquellas que hacen a la estrategia productivacomercial de la unidad de producción.

Frente a los cambios tecnológicos en el Cinturón Hortícola Platense en la década del 90 los asesores técnicos privados tomaron dimensiones relevantes de acuerdo a sus capacidades y conocimientos para afrontar este nuevo desafío. Su incidencia se manifiesta de forma mas clara en producciones de alto nivel tecnológico y asociados a planteos netamente empresariales.

La asociación entre variables tecnológicas productivas - comerciales identifican a tres grandes grupos de predios: unos a la vanguardia del modelo tecnológico, con asesoramiento profesional, con canales diferenciales de comercialización y lógicas típicamente empresariales; otros con estrategias tradicionales en cuanto a formas de producción y comercialización, sin asesoramiento técnico formal y un tercer grupo transicional entre los dos anteriores en cuanto a las variables mencionadas.

\section{REFERENCIAS}

1. Secretaria de Agricultura, Ganadería, Pesca y Alimentación Censo Hortícola del Cinturón Hortícola Bonaerense del Gran Buenos Aires 1998. Disponible en http:// www sagpya.mecon.gov.ar. Acceso en marzo de 2004.

2. BENENCIA, R. Nuevas formas de organización del trabajo rural en la Argentina. Su manifestación en la horticultura Bonaerense. Realidad Económica N 128. pp: 105-123, 1994.

3. HANG, G; BIFARETTI, A Y SARANDÓN R. Caracterización del sistema de producción hortícola empresario en el partido de La Plata Revista de la Facultad de Agronomía. Tomo 71 (2), 1995. 
4. SELIS, D. Efectos del cambio tecnológico sobre las condiciones de producción y reproducción del sector hortícola de La Plata. En RINGUELET, R. (Coord) Espacio tecnológico, población y reproducción social en el Sector Hortícola de La Plata. Serie Estudios/Investigación. Facultad de Humanidades y Cs. De la Educación, UNLP, 2000. P. 89-116.

5. ARCHENTI, A; RINGUELET, R; SALVA, M.C. Los procesos de diferenciación de los productores hortícolas de La Plata. Continuidad y Cambio. Revista ETNIA № 38-39. Olavarria, Pp: 57-63, 1993.

6. HANG, G. Y BIFARETTI, A. Sector Hortícola Platense. Realidad Económica № 131. Pp, 72-97, 1995

7. BOCHETTO, R. M. Marco Conceptual para caracterizar Sistemas Reales de Producción Agropecuaria, asociado al proceso de cambio tecnológico. En: VIII REUNIÓN ANUAL DE LAASOCIACIÓN ARGENTINA DE ECONOMÍAAGRARIA. Bs As. En actas. 30 pp, Nov. 1977.

8. CIEZA, R. El rol de los Ingenieros Agrónomos en el cambio tecnológico hortícola del Gran La Plata. En: III JORNADAS INTERDISCIPLINARIAS DE ESTUDIOS AGRARIOS Y AGROINDUSTRIALES. Facultad de Ciencias Económicas, PIEAIIHES. Bs. As. En actas de CD. 23 pp, 2003.

9. BENENCIA, R. La horticultura bonaerense. Desarrollo Económico. Vol. 34 № 133. Pp 83-97, 1994.

10. CRISCI, J.V Y LOPEZ AMENGOL, M.F. Introducción a la teoría y práctica de la taxonomía numerica. Secretaria General de la OEA. Programa regional de desarrollo científico y tecnológico Washington D.C. Serie de Biología, 1983. 132 p. (Monografía $\left.\mathrm{N}^{\circ} 26\right)$

11. SOKAL, R. Y MICHENER, R. A statistical metod for evaluating systematic relationships. University Kansas Sci.Bull. 38. Pp 1409-1438, 1958.

Recebido em 02/11/2004 Aceito em 04/05/2005 